\title{
The effects of antenatal parental breastfeeding education and counseling on the duration of breastfeeding, and maternal and paternal attachment
}

\author{
Zeliha Burcu Yurtsal ${ }^{*}$, Gulay Kocoglu ${ }^{2}$ \\ ${ }^{1}$ Cumhuriyet UniversityFaculty of Health Sciences, Department of Midwifery, Sivas, Turkey \\ ${ }^{2}$ Cumhuriyet University Faculty of Medicine, Department of Public Health, Sivas, Turkey
}

\begin{abstract}
Background: Although all health benefits of breastfeeding are well established for mothers and newborns, the rates of exclusive breastfeeding of infants are lower than expected among Turkish mothers. Objectives: This research was conducted to investigate the effects of training and counseling services given to primiparous women and their spouses on the breastfeeding process, and maternal-paternal attachment.

Methods: The research was conducted in two Hospitals in Sivas, a province in Turkey. Accordingly, 76 women and their spouses were separated randomly as "experimental" and "control" groups. Descriptive statistical methods, parametric and non-parametric tests were used in the statistical analysis of the data.

Results: According to the data, the statistical difference between the two groups was not significant in terms of socio-demographic characteristics of pregnant women and their spouses $(p>0.05)$. It was determined that while $94.7 \%$ of the experimental-group exclusively breastfed their babies, only $26.3 \%$ of the control-group mothers exclusively breastfed their babies for the first 6 months postpartum $(\mathrm{p}<0.05)$. The statistical difference between the two groups was significant $(\mathrm{p}<0.05)$. The rate of exclusive breastfeeding within the first 6 months postpartum among the participants given breastfeeding training was 50.4 times higher than that among those who were not given training. (Odds:50.400, \%95 CI).

Conclusion: In conclusion, in the present study, it was determined that the rates for the mothers' early initiation of breastfeeding, exclusive breastfeeding continuation rates and the mean scores the participants obtained from mean scale scores increased when they were given training and counselling on breastfeeding from the prenatal period until the end of the first sixth month postpartum.
\end{abstract}

\section{Introduction}

Varies organizations, including the World Health Organization and the American Academy of Pediatrics, recommend exclusive breastfeeding for the first six months of life [1,2]. Although breastfeeding is a common behavior in Turkey, the rates for the exclusive breastfeeding of babies are low $[3,4]$. The exclusive breastfeeding rate across Turkey during the first 6 months postpartum is $30.1 \%$ [5].

Exclusive breastfeeding of babies holds an important place within the basic health services. Today, basic health care requires that individuals should take on health care responsibilities for protecting and promoting their own health. It is also emphasized that health education plays an important part in one's acquiring self-care responsibilities [6]. Therefore, mothers should be informed and supported about breastfeeding [7].

Some of the important factors affecting the duration of breastfeeding are reported to be psychosocial factors such as mothers' determination to breastfeed, fathers' support on this issue and mothers' self-confidence. Given the role of fathers in the family regarding decision-making, it is important to include them in training programs so that they support their wives in breastfeeding [8].

Attachment theory refers to secure attachment bonds developing between an infant and a parent when the baby sends a signal giving clues of its needs and the adult caregiver responds this signal. In recent years, attachment theory has been developed to include the fathers [9].

This present study was designed as an intervention study and conducted to investigate the effect of breastfeeding training and counseling services given to both parents between the last trimester of pregnancy and six months postpartum on the breastfeeding process and maternal-paternal attachment.

\section{Materials and methods}

\section{The study population}

Primiparous pregnant women who presented to the pregnancy outpatient clinics of two hospitals in Sivas between May 1, 2013 and August 11, 2013 and their husbands comprised the study population.

Correspondence to: Dr. Zeliha Burcu YURTSAL, Cumhuriyet University Faculty of Health Sciences, Department of Midwifery, 58140, Sivas, Turkey, Tel: 0905323378084, Fax: 0903462191261, E-mail: burcuyurtsal@hotmail.com

Key words: human breast milk, exclusive breastfeeding, maternal and paternal attachment

Received: September 13, 2015; Accepted: October 02, 2015; Published: October 05, 2015 


\section{Sampling design and Sample size}

In this intervention study, given the values of $\alpha=0.05, \beta=0.20$, (1$\beta)=0.80$, it was decided to assign 38 persons to each group, and the test power was found as $\mathrm{P}=0.80063$. When the pregnant women were randomly assigned to both groups, the random number table was used. Therefore, 76 pregnant women and their husbands who met the inclusion criteria and agreed to participate in the study were randomly assigned to the "experimental" and "control" groups.

The inclusion criteria of the study were as follows: having at least primary school education, having no communication problem (language, speech and hearing problems, etc.), agreeing to participate in the study with her husband, having no previous breastfeeding experience, residing in the city center of Sivas, volunteering to participate in the study, having a nuclear family, being a housewife or not having to work for 6 months after delivery, being $\geq 35$-week pregnant, being primiparous, giving birth at term, mothers with healthy fetus, having no obstacles to breastfeeding during the postpartum period, having no previous training or counseling on breastfeeding.

\section{Tools used for collecting data}

The study data were collected with four questionnaires developed by the researcher through reviewing the pertinent literature [1013]: the Questionnaire for Prospective Mothers (Appendix 1), the Questionnaire for Prospective Fathers (Appendix 2), the Postpartum Information Form (Appendix 3) and the Follow-up Form Concerning Breastfeeding Characteristics "(Appendix 4). The form called IMDAT, an acronym of a Turkish scale which means "Scoring System for the Amount of Breast Milk Received by a Baby", was developed by Yigit and Arslan to assess the amount of mother's milk that the baby receives. While the 10-point full score indicates that baby receives human breast milk at the best level, points 7 or lower indicate that the baby does not receive enough breast milk [14].

"Postnatal Breastfeeding Self-Efficacy Scale-Short Form" developed by Dennis and Faux was adapted into Turkish by Tokat [15]. The lowest and highest possible scores to be obtained from the scale were 14 and 70 respectively. The higher the score is, the higher the breastfeeding self-efficacy is.

The Maternal Attachment Scale was developed by Mary E. Muller. The Turkish version of the scale was developed by Kavlak and Şirin. The lowest and highest possible scores to be obtained from the scale are 26 and 104 respectively [16,17].

The Postnatal Paternal-Infant Attachment Questionnaire [18] developed by John T. Condon was adapted into Turkish by Güleç. The scale is interpreted through the total and subscale scores. High scores indicate that attachment is high [19].

\section{Training process}

During the training process, the "Breastfeeding Counselling Manual" and the cassette "the Best Food for a Baby is its Mother's Milk" prepared by the Ministry of Health were used. The parents watched the training cassette for about half an hour. Breastfeeding training was given using the question-response method accompanied with written materials, and it took about an hour. Breastfeeding positions were taught to pregnant women and their spouses using the demonstration technique on baby mannequins. How to manually express their breastmilk was taught to all mothers. The parents' questions on the training, childbirth and infant care were answered.

\section{Use of tools for collecting data}

Random selection was used to assign the participants to the experimental and control groups using the one-to-one matching method taking their educational status and delivery modes into account.

The experimental group; $1^{\text {st }}$ interview in the hospital: having meetings with the participants before the delivery and determination of the training schedule,

$\mathbf{1}^{\text {st }}$ home visit: prenatal implementation of appendices 1 and 2, providing training for the parents on how to breastfeed in line with the training process,

$2^{\text {nd }}$ interview in the hospital: continuation of training and counseling on breastfeeding within the first 24 hours postpartum; implementation of appendices 3, 4 and IMDAT,

$2^{\text {nd }}$ home visit: repeating the content of the training, and breastfeeding skills at the end of the $1^{\text {st }}$ week postpartum; implementation of appendix 4,

$3^{\text {rd }}$ home visit: continuation of training and counseling on breastfeeding at the end of the $2^{\text {nd }}$ week postpartum; implementation of appendix 4 ,

$4^{\text {th }}$ home visit: continuation of training and counseling on breastfeeding at the end of $3^{\text {rd }}$ week postpartum; implementation of appendix 4,

$5^{\text {th }}$ home visit: continuation of training and counseling on breastfeeding at the end of the $1^{\text {st }}$ month postpartum; implementation of appendix 4 ,

Interviews on the phone; $1^{\text {st }}$ interview: continuation of training and counseling on breastfeeding at the end of the $2^{\text {nd }}$ month postpartum; implementation of appendix 4,

$2^{\text {nd }}$ interview: continuation of training and counseling on breastfeeding at the end of the $3^{\text {rd }}$ month postpartum; implementation of appendix 4 ,

$3^{\text {rd }}$ interview: continuation of training and counseling on breastfeeding at the end of the $4^{\text {th }}$ month postpartum; implementation of appendix 4 ,

$4^{\text {th }}$ interview on the phone: continuation of training and counseling on breastfeeding at the end of the $5^{\text {th }}$ month postpartum; implementation of appendix 4,

$6^{\text {th }}$ home visit: at the end of the $6^{\text {th }}$ month postpartum, continuation of training and counseling on breastfeeding; implementation of appendix 4 and implementation of the Self-efficacy Scale, IMDAT, Maternal Attachment Scale and Paternal Attachment Scale, and the final assessment.

The control group; $1^{\text {st }}$ interview in the hospital: having meetings with the participants within the first 24 hours postpartum and implementation of appendices 1,2,3,4 and IMDAT,

$1^{\text {st }}$ interview on the phone: at the end of the $6^{\text {th }}$ month postpartum, implementation of appendix 4 and implementation of the Self-efficacy Scale, IMDAT, Maternal Attachment Scale and Paternal Attachment Scale, and the final assessment.

\section{Statistical analysis}

The data obtained from the study were loaded into the SPSS 
(Statistical Package for Social Sciences) and evaluated with the significance of the difference between the two means when the parametric test assumptions were fulfilled and with the Chi-square test and Fisher's exact test, Fisher's exact Chi-square test when the parametric test assumptions were not fulfilled. The error level was taken as 0.05 .

\section{Results}

The study comprised 76 pregnant women and 76 spouses. While $55.3 \%$ of the pregnant women in the experimental group were between the ages of 25 and 33, this rate in the control group was $47.4 \%$. In both groups, $47.4 \%$ of the pregnant women were university graduates, and $65.8 \%$ of the women in the experimental group and $60.5 \%$ of the women in the control group were employed. All of the women in the experimental group and $97.4 \%$ of the women in the control group had health insurance. While the rate of those with an average monthly income over $\$ 1000$ was $50.5 \%$ in the experimental group, the rate was $44.7 \%$ in the control group. For $89.5 \%$ of the women in both groups, the longest place of residence was a city center.

As to the spouses, $92.1 \%$ in the experimental group and $86.8 \%$ in the control group were in the $25-36$ age group, and $52.6 \%$ in the experimental group and $47.4 \%$ in the control group were university graduates. All of the spouses in both groups were employed and almost half of them were workers. There were no significant differences between the two groups in terms of the sociodemographic characteristics of the pregnant women and their spouses $(\mathrm{p}>0.05)$.

Distribution of the pregnant women in terms of their knowledge and views on breastfeeding in the prenatal period is given in Table 1 . All of the women in both groups stated that they wanted to breastfeed; however, this was not included in the table. The analysis of the Table 1 shows that all of the women in the experimental group had positive and

Table 1. Distribution of the pregnant women in terms of their knowledge and views on breastfeeding in the prenatal period.

\begin{tabular}{|c|c|c|c|c|c|}
\hline \multirow[t]{2}{*}{ Characteristics } & \multicolumn{2}{|c|}{ Experimental group } & \multicolumn{2}{|c|}{ Control group } & \multirow[t]{2}{*}{ Statistical analysis } \\
\hline & Number & $\%$ & Number & $\%$ & \\
\hline $\begin{array}{l}\text { Duration of exclusive breastf } \\
\leq 5 \text { months } \\
6 \text { months }\end{array}$ & $\begin{array}{c}0 \\
38\end{array}$ & $\begin{array}{c}0.0 \\
100.0\end{array}$ & $\begin{array}{c}5 \\
33\end{array}$ & $\begin{array}{l}13.2 \\
86.8\end{array}$ & $\mathrm{p}=0.001^{*}$ \\
\hline $\begin{array}{l}\text { Total breastfeeding } \\
\text { Time/Age } \\
\leq 1.5 \text { years } \\
\geq 2 \text { years }\end{array}$ & $\begin{array}{c}0 \\
38\end{array}$ & $\begin{array}{c}0.0 \\
100.0\end{array}$ & $\begin{array}{l}16 \\
22\end{array}$ & $\begin{array}{l}42.1 \\
57.9\end{array}$ & $\begin{array}{c}\chi^{2}=20.26 \\
\mathrm{p}=0.001\end{array}$ \\
\hline $\begin{array}{l}\text { Having training on breast mi } \\
\text { Yes } \\
\text { No }\end{array}$ & $\begin{array}{c}38 \\
0\end{array}$ & $\begin{array}{c}100.0 \\
0.0\end{array}$ & $\begin{array}{c}3 \\
35\end{array}$ & $\begin{array}{c}7.9 \\
92.1\end{array}$ & $\begin{array}{c}\chi^{2}=64.87 \\
\mathrm{p}=0.001\end{array}$ \\
\hline $\begin{array}{l}\text { Initiation of breastfeeding } \\
\text { Within the first } 30 \text { minutes } \\
\text { Any time }\end{array}$ & $\begin{array}{c}38 \\
0\end{array}$ & $\begin{array}{c}100.0 \\
0.0\end{array}$ & $\begin{array}{c}3 \\
35\end{array}$ & $\begin{array}{c}7.9 \\
92.1\end{array}$ & $\begin{array}{c}\chi^{2}=64.87 \\
\mathrm{p}=0.001\end{array}$ \\
\hline $\begin{array}{l}\text { Duration of each breastfeedir } \\
5-10 \text { minutes } \\
15-20 \text { minutes } \\
\text { Does not know }\end{array}$ & $\begin{array}{c}0 \\
38 \\
0\end{array}$ & $\begin{array}{c}0.0 \\
100.0 \\
0.0\end{array}$ & $\begin{array}{c}2 \\
6 \\
30\end{array}$ & $\begin{array}{c}5.3 \\
15.8 \\
78.9\end{array}$ & $\begin{array}{c}\chi^{2}=68.40 \\
\mathrm{p}=0.001\end{array}$ \\
\hline $\begin{array}{l}\text { Benefits of breastfeeding for } \\
\text { Healthy baby } \\
\text { Strong immunity } \\
\text { Does not know }\end{array}$ & $\begin{array}{c}25 \\
13 \\
0\end{array}$ & $\begin{array}{c}65.8 \\
34.2 \\
0.0\end{array}$ & $\begin{array}{c}6 \\
13 \\
19\end{array}$ & $\begin{array}{l}15.8 \\
34.2 \\
50.0\end{array}$ & $\begin{aligned} \chi^{2} & =30.64 \\
\mathrm{p} & =0.001\end{aligned}$ \\
\hline $\begin{array}{l}\text { Is breastfeeding beneficial for } \\
\text { Yes } \\
\text { No }\end{array}$ & $\begin{array}{c}38 \\
0\end{array}$ & $\begin{array}{c}100.0 \\
0.0\end{array}$ & $\begin{array}{l}25 \\
13\end{array}$ & $\begin{array}{l}65.8 \\
34.2\end{array}$ & $\begin{array}{l}\chi^{2}=15.68 \\
\mathrm{p}=0.001\end{array}$ \\
\hline $\begin{array}{l}\text { Benefits of breastfeeding for } \\
\text { Infant-mother attachment } \\
\text { Losing weight easily } \\
\text { Protection from breast cancer } \\
\text { Recovery of the womb } \\
\text { Does not know }\end{array}$ & $\begin{array}{c}12 \\
9 \\
16 \\
1 \\
0\end{array}$ & $\begin{array}{c}31.6 \\
23.7 \\
42.1 \\
2.6 \\
0.0\end{array}$ & $\begin{array}{c}0 \\
13 \\
0 \\
0 \\
25\end{array}$ & $\begin{array}{c}0.0 \\
34.2 \\
0.0 \\
0.0 \\
65.8\end{array}$ & $\begin{array}{c}\chi^{2}=54.72 \\
\mathrm{p}=0.001\end{array}$ \\
\hline $\begin{array}{l}\text { Using a bottle } \\
\text { Yes } \\
\text { No }\end{array}$ & $\begin{array}{c}0 \\
38\end{array}$ & $\begin{array}{c}0.0 \\
100.0\end{array}$ & $\begin{array}{c}37 \\
1\end{array}$ & $\begin{array}{c}97.4 \\
2.6\end{array}$ & $\begin{array}{c}\chi^{2}=72.10 \\
\mathrm{p}=0.001\end{array}$ \\
\hline $\begin{array}{l}\text { Using a pacifier } \\
\text { Yes } \\
\text { No }\end{array}$ & $\begin{array}{c}0 \\
38\end{array}$ & $\begin{array}{c}0.0 \\
100.0\end{array}$ & $\begin{array}{c}37 \\
1\end{array}$ & $\begin{array}{c}97.4 \\
2.6\end{array}$ & $\begin{array}{c}\chi^{2}=72.10 \\
\mathrm{p}=0.001\end{array}$ \\
\hline $\begin{array}{l}\text { Having talks with the spouse } \\
\text { Yes } \\
\text { No }\end{array}$ & $\begin{array}{c}38 \\
0\end{array}$ & $\begin{array}{c}100.0 \\
0.0\end{array}$ & $\begin{array}{c}2 \\
36\end{array}$ & $\begin{array}{c}5.3 \\
94.7\end{array}$ & $\begin{array}{c}\chi^{2}=72.10 \\
\mathrm{p}=0.001\end{array}$ \\
\hline Total & 38 & 100.0 & 38 & 100.0 & \\
\hline
\end{tabular}

*Fisher Exact Test was implemented. 
accurate knowledge in terms of the variables regarding breastfeeding but that there were significant differences between the two groups when all the variables were taken into account $(\mathrm{p}<0.05)$.

Distribution of the pregnant women's spouses in terms of their knowledge and views on breastfeeding in the prenatal period is given in Table 2. Similarly, the analysis of the Table 2 shows that all of the spouses in the experimental group had positive and accurate knowledge in terms of the variables regarding breastfeeding but that there were significant differences between the two groups when all the variables were taken into account $(\mathrm{p}<0.05)$.

Distribution of the pregnant women in terms of their characteristics regarding breastfeeding in the intrapartum and postpartum periods is given in Table 3 . While $65.8 \%$ of the mothers in the experimental and control groups gave birth through normal vaginal delivery, $34.2 \%$ of them gave birth through cesarean section. In both groups, spinal anesthesia was administered to the mothers who gave birth through cesarean section. There were significant differences between the groups in terms of starting breastfeeding within the first 30 minutes postpartum and having problems during the first breastfeeding. The experimentalgroup mothers were determined to display more accurate and positive attitudes than did the control-group mothers $(\mathrm{p}<0.05)$.

Distribution of the pregnant women in terms of their characteristics regarding breastfeeding within the first 48 hours and 6 months postpartum is given in Table 4. The experimental-group mothers were determined to display more accurate and scientific attitudes than did the control-group mothers and the difference between the two groups was statistically significant $(\mathrm{p}<0.05)$.

Table 5 shows the data on the mothers' exclusive breastfeeding behaviors within the first six months postpartum. In the experimental group, while all the mothers exclusively breastfed their babies within the first 5 months postpartum, $94.7 \%$ of them continued exclusive breastfeeding in the $6^{\text {th }}$ month postpartum. In the control group, about $80 \%$ of the mothers practiced exclusive breastfeeding within the first three months postpartum; however, this rate declined to $26.3 \%$ at the end of the $6^{\text {th }}$ month postpartum. There were significant differences

Table 2. Distribution of the pregnant women's spouses in terms of their knowledge and views on breastfeeding in the prenatal period.

\begin{tabular}{|c|c|c|c|c|c|}
\hline \multirow[t]{2}{*}{ Characteristics } & \multicolumn{2}{|c|}{ Experimental group } & \multicolumn{2}{|c|}{ Control group } & \multirow{2}{*}{$\begin{array}{l}\text { Statistical } \\
\text { analysis }\end{array}$} \\
\hline & Number & $\%$ & Number & $\%$ & \\
\hline $\begin{array}{l}\text { Duration of exclusive breas } \\
\leq 5 \text { months } \\
6 \text { months }\end{array}$ & $\begin{array}{c}0 \\
38\end{array}$ & $\begin{array}{c}0.0 \\
100.0\end{array}$ & $\begin{array}{c}9 \\
29\end{array}$ & $\begin{array}{l}23.7 \\
76.3\end{array}$ & $\mathrm{p}=0.001^{*}$ \\
\hline $\begin{array}{l}\text { Total breastfeeding } \\
\text { Time/Age } \\
\leq 1.5 \text { years } \\
\geq 2 \text { years }\end{array}$ & $\begin{array}{c}0 \\
38\end{array}$ & $\begin{array}{c}0.0 \\
100.0\end{array}$ & $\begin{array}{l}20 \\
18\end{array}$ & $\begin{array}{l}52.6 \\
47.4\end{array}$ & $\begin{array}{c}\chi^{2}=27.14 \\
\mathrm{p}=0.001\end{array}$ \\
\hline $\begin{array}{l}\text { Having training on breast } \\
\text { Yes } \\
\text { No }\end{array}$ & $\begin{array}{c}38 \\
0\end{array}$ & $\begin{array}{c}100.0 \\
0.0\end{array}$ & $\begin{array}{c}0 \\
38\end{array}$ & $\begin{array}{c}0.0 \\
100.0\end{array}$ & $\begin{array}{c}\chi^{2}=76.00 \\
\mathrm{p}=0.001\end{array}$ \\
\hline $\begin{array}{l}\text { Initiation of breastfeeding } \\
\text { Within the first } 30 \text { minutes } \\
\text { Within the first hour } \\
\text { Any time } \\
\text { Does not know }\end{array}$ & $\begin{array}{c}38 \\
0 \\
0 \\
0\end{array}$ & $\begin{array}{c}100.0 \\
0.0 \\
0.0 \\
0.0\end{array}$ & $\begin{array}{c}0 \\
16 \\
2 \\
20\end{array}$ & $\begin{array}{c}0.0 \\
42.1 \\
5.3 \\
52.6\end{array}$ & $\begin{array}{c}\chi^{2}=76.00 \\
\mathrm{p}=0.001\end{array}$ \\
\hline $\begin{array}{l}\text { Duration of each breastfee } \\
5-10 \text { minutes } \\
15-20 \text { minutes } \\
\text { Does not know }\end{array}$ & $\begin{array}{c}0 \\
38 \\
0\end{array}$ & $\begin{array}{c}0.0 \\
100.0 \\
0.0\end{array}$ & $\begin{array}{c}0 \\
7 \\
31\end{array}$ & $\begin{array}{c}0.0 \\
18.4 \\
81.6\end{array}$ & $\begin{array}{c}\chi^{2}=76.00 \\
\mathrm{p}=0.001\end{array}$ \\
\hline $\begin{array}{l}\text { Benefits of breastfeeding fo } \\
\text { Healthy baby } \\
\text { Strong immunity } \\
\text { Does not know }\end{array}$ & $\begin{array}{c}25 \\
13 \\
0\end{array}$ & $\begin{array}{c}65.8 \\
34.2 \\
0.0\end{array}$ & $\begin{array}{c}14 \\
1 \\
23\end{array}$ & $\begin{array}{c}36.8 \\
2.6 \\
60.5\end{array}$ & $\begin{array}{c}\chi^{2}=36.38 \\
\mathrm{p}=0.001\end{array}$ \\
\hline $\begin{array}{l}\text { Is breastfeeding beneficial } \\
\text { Yes } \\
\text { No }\end{array}$ & $\begin{array}{c}38 \\
0\end{array}$ & $\begin{array}{c}100.0 \\
0.0\end{array}$ & $\begin{array}{l}21 \\
17\end{array}$ & $\begin{array}{l}55.3 \\
44.7\end{array}$ & $\begin{aligned} \chi^{2} & =21.89 \\
\mathrm{p} & =0.001\end{aligned}$ \\
\hline $\begin{array}{l}\text { Using a bottle } \\
\text { Yes } \\
\text { No }\end{array}$ & $\begin{array}{c}0 \\
38\end{array}$ & $\begin{array}{c}0.0 \\
100.0\end{array}$ & $\begin{array}{c}38 \\
0\end{array}$ & $\begin{array}{c}100.0 \\
0.0\end{array}$ & $\begin{array}{l}\chi^{2}=72.10 \\
p=0.001\end{array}$ \\
\hline $\begin{array}{l}\text { Using a pacifier } \\
\text { Yes } \\
\text { No }\end{array}$ & $\begin{array}{c}0 \\
38 \\
\end{array}$ & $\begin{array}{c}0.0 \\
100.0\end{array}$ & $\begin{array}{c}38 \\
0\end{array}$ & $\begin{array}{c}100.0 \\
0.0\end{array}$ & $\begin{array}{c}\chi^{2}=76.00 \\
\mathrm{p}=0.001\end{array}$ \\
\hline $\begin{array}{l}\text { Having talks with the spou } \\
\text { Yes } \\
\text { No }\end{array}$ & $\begin{array}{c}38 \\
0\end{array}$ & $\begin{array}{c}100.0 \\
0.0\end{array}$ & $\begin{array}{c}2 \\
36\end{array}$ & $\begin{array}{c}5.3 \\
94.7\end{array}$ & $\begin{array}{c}\chi^{2}=76.00 \\
p=0.001\end{array}$ \\
\hline Total & 38 & 100.0 & 38 & 100.0 & \\
\hline
\end{tabular}

*Fisher Exact Test was implemented. 
Yurtsal ZB (2015) The effects of antenatal parental breastfeeding education and counseling on the duration of breastfeeding, and maternal and paternal attachment

Table 3. Distribution of the mothers' in terms of their characteristics regarding breastfeeding in the intrapartum and postpartum periods.

\begin{tabular}{|c|c|c|c|c|c|}
\hline \multirow[t]{2}{*}{ Characteristics } & \multicolumn{2}{|c|}{ Experimental group } & \multicolumn{2}{|c|}{ Control group } & \multirow{2}{*}{$\begin{array}{c}\text { Statistical } \\
\text { analysis }\end{array}$} \\
\hline & Number & $\%$ & Number & $\%$ & \\
\hline $\begin{array}{l}\text { Delivery method } \\
\text { Normal vaginal birth } \\
\text { Cesarean section }\end{array}$ & $\begin{array}{l}25 \\
13\end{array}$ & $\begin{array}{l}65.8 \\
34.2\end{array}$ & $\begin{array}{l}25 \\
13\end{array}$ & $\begin{array}{l}65.8 \\
34.2\end{array}$ & $\mathrm{p}=1.000^{*}$ \\
\hline $\begin{array}{l}\text { Baby's gender } \\
\text { Girl } \\
\text { Boy }\end{array}$ & $\begin{array}{l}21 \\
17\end{array}$ & $\begin{array}{l}55.3 \\
44.7\end{array}$ & $\begin{array}{l}20 \\
18\end{array}$ & $\begin{array}{l}52.6 \\
47.4\end{array}$ & $\begin{array}{l}\chi^{2}=0.053 \\
\mathrm{p}=0.818\end{array}$ \\
\hline $\begin{array}{l}\text { Time for the first breastfeeding } \\
\text { The first } 15 \text { minutes } \\
\text { The first } 30 \text { minutes } \\
\text { The first } 1 \text { hour } \\
\text { Between } 2-24 \text { hours }\end{array}$ & $\begin{array}{c}36 \\
2 \\
0 \\
0\end{array}$ & $\begin{array}{c}94.7 \\
5.3 \\
0.0 \\
0.0\end{array}$ & $\begin{array}{c}1 \\
0 \\
19 \\
18\end{array}$ & $\begin{array}{c}2.6 \\
0.0 \\
50.0 \\
47.4\end{array}$ & $\begin{array}{l}\chi^{2}=72.10 \\
p=0.001\end{array}$ \\
\hline $\begin{array}{l}\text { Problems in the first lactation } \\
\text { Yes } \\
\text { No }\end{array}$ & $\begin{array}{c}7 \\
31\end{array}$ & $\begin{array}{l}18.4 \\
81.6\end{array}$ & $\begin{array}{c}29 \\
9\end{array}$ & $\begin{array}{l}76.3 \\
23.7\end{array}$ & $\begin{array}{l}\chi^{2}=25.54 \\
\mathrm{p}=0.001\end{array}$ \\
\hline $\begin{array}{l}\text { The person who helped with the firs } \\
\text { breastfeeding } \\
\text { The researcher } \\
\text { Mother and mother-in- law }\end{array}$ & $\begin{array}{c}38 \\
0\end{array}$ & $\begin{array}{c}100.0 \\
0.0\end{array}$ & $\begin{array}{c}0 \\
38\end{array}$ & $\begin{array}{c}0.0 \\
100.0\end{array}$ & $\begin{array}{c}\chi^{2}=76.00 \\
\mathrm{p}=0.001\end{array}$ \\
\hline $\begin{array}{l}\text { Complementary food besides breast milk } \\
\text { Yes } \\
\text { No }\end{array}$ & $\begin{array}{c}0 \\
38\end{array}$ & $\begin{array}{c}0.0 \\
100.0\end{array}$ & $\begin{array}{l}19 \\
19\end{array}$ & $\begin{array}{l}50.0 \\
50.0\end{array}$ & $\begin{array}{l}\chi^{2}=25.33 \\
\mathrm{p}=0.001\end{array}$ \\
\hline $\begin{array}{l}\text { Feeling that she is competent in breastfeeding } \\
\text { Yes } \\
\text { No }\end{array}$ & $\begin{array}{c}38 \\
0\end{array}$ & $\begin{array}{c}100.0 \\
0.0\end{array}$ & $\begin{array}{c}2 \\
36\end{array}$ & $\begin{array}{c}5.3 \\
94.7\end{array}$ & $\begin{array}{l}\chi^{2}=68.40 \\
\mathrm{p}=0.001\end{array}$ \\
\hline Total & 38 & 100.0 & 38 & 100.0 & \\
\hline
\end{tabular}

*Fisher Exact Test was implemented.

Table 4. Distribution of the mothers' in terms of their characteristics regarding breastfeeding within the first 48 hours and 6 months postpartum.

\begin{tabular}{|c|c|c|c|c|c|c|c|c|c|c|}
\hline \multirow{3}{*}{ Characteristics } & \multicolumn{5}{|c|}{ First 48 hours postpartum } & \multicolumn{5}{|c|}{ End of the $6^{\text {th }}$ month postpartum } \\
\hline & \multicolumn{2}{|c|}{ Experimental group } & \multicolumn{2}{|c|}{ Control group } & \multirow{2}{*}{$\begin{array}{c}\text { Statistical } \\
\text { analysis }\end{array}$} & \multicolumn{2}{|c|}{ Experimental group } & \multicolumn{2}{|c|}{ Control group } & \multirow{2}{*}{$\begin{array}{c}\text { Statistical } \\
\text { analysis }\end{array}$} \\
\hline & Number & $\%$ & Number & $\%$ & & Number & $\%$ & Number & $\%$ & \\
\hline $\begin{array}{l}\text { Breastfeeding Frequency } \\
1 \text { hour } \\
2 \text { hours } \\
\text { not breastfeeding }\end{array}$ & $\begin{array}{c}5 \\
33 \\
0\end{array}$ & $\begin{array}{c}13.2 \\
86.8 \\
0.0\end{array}$ & $\begin{array}{c}24 \\
14 \\
0\end{array}$ & $\begin{array}{c}63.2 \\
36.8 \\
0.0\end{array}$ & $\begin{array}{c}\chi^{2}=20.12 \\
\mathrm{p}=0.001\end{array}$ & $\begin{array}{c}1 \\
37 \\
0\end{array}$ & $\begin{array}{c}2.6 \\
97.4 \\
0.0\end{array}$ & $\begin{array}{c}10 \\
27 \\
1\end{array}$ & $\begin{array}{l}26.3 \\
71.1 \\
2.6\end{array}$ & $\mathrm{p}=0.001^{*}$ \\
\hline $\begin{array}{l}\text { Duration of each breastfeeding } \\
5-10 \text { minutes } \\
15-20 \text { minutes } \\
\text { not breastfeeding }\end{array}$ & $\begin{array}{c}1 \\
37 \\
0\end{array}$ & $\begin{array}{c}2.6 \\
97.4 \\
0.0\end{array}$ & $\begin{array}{c}34 \\
4 \\
0\end{array}$ & $\begin{array}{c}89.5 \\
10.5 \\
0.0\end{array}$ & $\begin{aligned} \chi^{2} & =57.67 \\
\mathrm{p} & =0.001\end{aligned}$ & $\begin{array}{c}0 \\
38 \\
0\end{array}$ & $\begin{array}{c}0.0 \\
100.0 \\
0.0\end{array}$ & $\begin{array}{c}12 \\
25 \\
1\end{array}$ & $\begin{array}{c}31.6 \\
65.8 \\
2.6\end{array}$ & $\mathrm{p}=0.001^{*}$ \\
\hline $\begin{array}{l}\text { Continuation of breastfeeding } \\
\text { Yes } \\
\text { No }\end{array}$ & $\begin{array}{c}38 \\
0\end{array}$ & $\begin{array}{c}100.0 \\
0.0\end{array}$ & $\begin{array}{c}38 \\
0\end{array}$ & $\begin{array}{c}100.0 \\
0.0\end{array}$ & & $\begin{array}{c}38 \\
0\end{array}$ & $\begin{array}{c}100.0 \\
0.0\end{array}$ & $\begin{array}{c}35 \\
3 \\
\end{array}$ & $\begin{array}{c}92.1 \\
7.9\end{array}$ & $\mathrm{p}=0.001^{*}$ \\
\hline $\begin{array}{l}\text { Starting complementary food } \\
\text { Yes } \\
\text { No }\end{array}$ & $\begin{array}{c}0 \\
38\end{array}$ & $\begin{array}{c}0.0 \\
100.0\end{array}$ & $\begin{array}{l}21 \\
17 \\
\end{array}$ & $\begin{array}{l}55.3 \\
44.7\end{array}$ & $\begin{array}{l}\chi^{2}=29.01 \\
\mathrm{p}=0.001\end{array}$ & $\begin{array}{c}2 \\
36\end{array}$ & $\begin{array}{c}5.3 \\
94.7\end{array}$ & $\begin{array}{l}28 \\
10\end{array}$ & $\begin{array}{l}73.7 \\
26.3\end{array}$ & $\begin{array}{l}\chi^{2}=37.22 \\
\mathrm{p}=0.001\end{array}$ \\
\hline $\begin{array}{l}\text { Using a bottle } \\
\text { Yes } \\
\text { No }\end{array}$ & $\begin{array}{c}0 \\
38\end{array}$ & $\begin{array}{c}0.0 \\
100.0\end{array}$ & $\begin{array}{l}16 \\
22\end{array}$ & $\begin{array}{l}42.1 \\
57.9 \\
\end{array}$ & $\begin{array}{l}\chi^{2}=20.26 \\
\mathrm{p}=0.001\end{array}$ & $\begin{array}{c}0 \\
38\end{array}$ & $\begin{array}{c}0.0 \\
100.0\end{array}$ & $\begin{array}{c}30 \\
8\end{array}$ & $\begin{array}{l}78.9 \\
21.1\end{array}$ & $\begin{array}{c}\chi^{2}=49.56 \\
\mathrm{p}=0.001\end{array}$ \\
\hline $\begin{array}{l}\text { Using a pacifier } \\
\text { Yes } \\
\text { No }\end{array}$ & $\begin{array}{c}0 \\
38 \\
\end{array}$ & $\begin{array}{c}0.0 \\
100.0\end{array}$ & $\begin{array}{c}0 \\
38 \\
\end{array}$ & $\begin{array}{c}0.0 \\
100.0\end{array}$ & & $\begin{array}{c}0 \\
38\end{array}$ & $\begin{array}{c}0.0 \\
100.0\end{array}$ & $\begin{array}{c}35 \\
3\end{array}$ & $\begin{array}{c}92.1 \\
7.9\end{array}$ & $\begin{array}{l}\chi^{2}=64.87 \\
\mathrm{p}=0.001\end{array}$ \\
\hline $\begin{array}{l}\text { Breast problems } \\
\text { Yes } \\
\text { No }\end{array}$ & $\begin{array}{l}18 \\
20\end{array}$ & $\begin{array}{l}47.4 \\
52.6\end{array}$ & $\begin{array}{c}38 \\
0\end{array}$ & $\begin{array}{c}100.0 \\
0.0\end{array}$ & $\begin{array}{l}\chi^{2}=27.14 \\
p=0.001\end{array}$ & $\begin{array}{c}0 \\
38\end{array}$ & $\begin{array}{c}0.0 \\
100.0\end{array}$ & $\begin{array}{c}0 \\
38\end{array}$ & $\begin{array}{c}0.0 \\
100.0\end{array}$ & \\
\hline Total & 38 & 100.0 & 38 & 100.0 & & 38 & 100.0 & 38 & 100.0 & \\
\hline
\end{tabular}

*Fisher Exact Test was implemented.

between the two groups in terms of exclusive breastfeeding rates $(\mathrm{p}<0.05)$.

In Table 6, the mean scores obtained from the Breastfeeding Self-
Efficacy, IMDAT, Maternal Attachment and Paternal Attachment scales are given. The mean scores achieved by the experimental-group participants were higher than those achieved by the control-group participants. The difference between the two groups was significant $(\mathrm{P}<0.05)$. 
Table 5. Duration of mothers' exclusive breastfeeding $(n=76)$.

\begin{tabular}{|c|c|c|c|c|c|}
\hline \multirow[t]{2}{*}{ Characteristics } & \multicolumn{2}{|c|}{ Experimental group } & \multicolumn{2}{|c|}{ Control group } & \multirow{2}{*}{$\begin{array}{c}\text { Statistical } \\
\text { analysis }\end{array}$} \\
\hline & Number & $\%$ & Number & $\%$ & \\
\hline $\begin{array}{l}\text { First } 2 \text { days } \\
\text { Yes } \\
\text { No }\end{array}$ & $\begin{array}{c}38 \\
0\end{array}$ & $\begin{array}{c}100.0 \\
0.0\end{array}$ & $\begin{array}{l}17 \\
21\end{array}$ & $\begin{array}{l}44.7 \\
55.3\end{array}$ & $\begin{array}{l}\chi^{2}=29.01 \\
\mathrm{p}=0.001\end{array}$ \\
\hline \begin{tabular}{|l} 
week \\
Yes \\
No \\
\end{tabular} & $\begin{array}{c}38 \\
0\end{array}$ & $\begin{array}{c}100.0 \\
0.0\end{array}$ & $\begin{array}{c}34 \\
4\end{array}$ & $\begin{array}{l}89.5 \\
10.5\end{array}$ & $\mathrm{p}=0.115^{*}$ \\
\hline $\begin{array}{l}2 \text { weeks } \\
\text { Yes } \\
\text { No }\end{array}$ & $\begin{array}{c}38 \\
0\end{array}$ & $\begin{array}{c}100.0 \\
0.0\end{array}$ & $\begin{array}{c}33 \\
5\end{array}$ & $\begin{array}{l}86.8 \\
13.2\end{array}$ & $\mathrm{p}=0.054^{*}$ \\
\hline $\begin{array}{l}3 \text { weeks } \\
\text { Yes } \\
\text { No }\end{array}$ & $\begin{array}{c}38 \\
0\end{array}$ & $\begin{array}{c}100.0 \\
0.0\end{array}$ & $\begin{array}{c}33 \\
5\end{array}$ & $\begin{array}{l}86.8 \\
13.2\end{array}$ & $\mathrm{p}=0.054^{*}$ \\
\hline $\begin{array}{l}1 \text { month } \\
\text { Yes } \\
\text { No }\end{array}$ & $\begin{array}{c}38 \\
0\end{array}$ & $\begin{array}{c}100.0 \\
0.0\end{array}$ & $\begin{array}{c}33 \\
5\end{array}$ & $\begin{array}{l}86.8 \\
13.2\end{array}$ & $\mathrm{p}=0.054^{*}$ \\
\hline $\begin{array}{l}2 \text { months } \\
\text { Yes } \\
\text { No }\end{array}$ & $\begin{array}{c}38 \\
0\end{array}$ & $\begin{array}{c}100.0 \\
0.0\end{array}$ & $\begin{array}{c}33 \\
5\end{array}$ & $\begin{array}{l}86.8 \\
13.2\end{array}$ & $\mathrm{p}=0.054^{*}$ \\
\hline $\begin{array}{l}\text { 3months } \\
\text { Yes } \\
\text { No }\end{array}$ & $\begin{array}{c}38 \\
0\end{array}$ & $\begin{array}{c}100.0 \\
0.0\end{array}$ & $\begin{array}{c}31 \\
7\end{array}$ & $\begin{array}{l}81.6 \\
18.4\end{array}$ & $\mathrm{p}=0.001 *$ \\
\hline $\begin{array}{l}\text { 4months } \\
\text { Yes } \\
\text { No }\end{array}$ & $\begin{array}{c}38 \\
0\end{array}$ & $\begin{array}{c}100.0 \\
0.0\end{array}$ & $\begin{array}{l}21 \\
17\end{array}$ & $\begin{array}{l}55.3 \\
44.7\end{array}$ & $\begin{array}{l}\chi^{2}=21.89 \\
p=0.001\end{array}$ \\
\hline $\begin{array}{l}\text { 5months } \\
\text { Yes } \\
\text { No }\end{array}$ & $\begin{array}{c}38 \\
0\end{array}$ & $\begin{array}{c}100.0 \\
0.0\end{array}$ & $\begin{array}{l}10 \\
28\end{array}$ & $\begin{array}{l}26.3 \\
73.7\end{array}$ & $\begin{array}{l}\chi^{2}=44.33 \\
p=0.001\end{array}$ \\
\hline $\begin{array}{l}\text { 6months } \\
\text { Yes } \\
\text { No }\end{array}$ & $\begin{array}{c}36 \\
2\end{array}$ & $\begin{array}{c}94.7 \\
5.3\end{array}$ & $\begin{array}{l}10 \\
28\end{array}$ & $\begin{array}{l}26.3 \\
73.7\end{array}$ & $\begin{array}{l}\chi^{2}=37.22 \\
p=0.001\end{array}$ \\
\hline Total & 38 & 100.0 & 38 & 100.0 & \\
\hline
\end{tabular}

*Fisher Exact Test was implemented.

Table 6. Mean scores obtained from the Breastfeeding Self-Efficacy, IMDAT Maternal Attachment and Paternal Attachment Scales.

\begin{tabular}{|c|c|c|c|}
\hline \multirow[t]{2}{*}{ Scale scores } & Experimental group $(n=38)$ & $\begin{array}{c}\text { Control group } \\
(n=38)\end{array}$ & \multirow[t]{2}{*}{$\begin{array}{c}\text { Statistical } \\
\text { analysis }\end{array}$} \\
\hline & $\mathbf{X} \pm \mathrm{S}$ & $\mathbf{X} \pm \mathrm{S}$ & \\
\hline Breastfeeding Self-Efficacy & $69.14 \pm 1.5$ & $53.87 \pm 13.52$ & $\begin{array}{c}\mathrm{t}=6.91 \\
\mathrm{P}=0.001\end{array}$ \\
\hline IMDAT & $9.66 \pm 0.48$ & $7.87 \pm 1.68$ & $\begin{array}{c}\mathrm{t}=6.32 \\
\mathrm{P}=0.001\end{array}$ \\
\hline Maternal Attachment & $103.37 \pm 1.32$ & $83.16 \pm 12.99$ & $\begin{array}{c}\mathrm{t}=9.54 \\
\mathrm{P}=0.001\end{array}$ \\
\hline Paternal Attachment & $89.0 \pm 1.93$ & $72.26 \pm 9.70$ & $\begin{array}{l}\mathrm{t}=10.43 \\
\mathrm{P}=0.001\end{array}$ \\
\hline
\end{tabular}

The rate of exclusive breastfeeding within the first 6 months postpartum among the participants given breastfeeding training was 50.4 times higher than that among those who were not given training. (Odds:50.400, \%95 CI).

\section{Discussion}

While rates for breastfeeding for some period of time are high both in developed countries and in developing countries, exclusive breastfeeding rates are lower. According to the statistics released by the WHO in 2011, exclusive breastfeeding rate all over the world is $36 \%$ [20]. According to Turkey Demographic and Health Survey(TDHS)
2013, 96.0\% of the babies are breastfed for some period of time; however, an exclusive breastfeeding rate during the first six months of life is $30.1 \%$ [5]. In Ünsal et al.'s study, exclusive breastfeeding rates at birth and in the $1^{\text {st }}, 4^{\text {th }}$ and $6^{\text {th }}$ months postpartum are $97 \%, 91.5 \%, 46 \%$ and $8.7 \%$ respectively [21]. In the present study, $94.7 \%$ of the infants in the experimental group and $26.3 \%$ of the infants in the control group were exclusively breastfed in the $6^{\text {th }}$ month postpartum. The rates were $100.0 \%$ in the experimental group and $55.3 \%$ in the control group in the fourth month. The comparison of our results with those in the literature indicates that both breastfeeding initiation rates and continuation rates in the present study are higher than those in other 
studies. The high rates in the experimental group would undoubtedly be attributed to training given to pregnant women and their spouses in this group.

It is stated that exclusive breastfeeding rates increase if mothers are given breastfeeding training and supported after birth by medical staff [22-24]. In Üstüner and Bodur's study, exclusive breastfeeding rates among mothers who received breastfeeding training and were visited at home during the six months after birth were $64.0 \%$ in the first month, 23.0 in the second month and $9.0 \%$ in the sixth month [25]. These rates are much lower than the rates in the experimental group in the present study; thus, it can be said that providing training for both parents contributed to this difference.

It is reported that training on breast milk and breastfeeding should begin during the prenatal period. Pregnant women should be informed about the benefits and methods of breastfeeding with prenatal training [26-28]. Several studies have revealed that starting training on breastfeeding during pregnancy affects the increases exclusive breastfeeding rates [29-31]. In Onbaşı et al.'s study, it was determined that exclusive breastfeeding rates were higher among mothers who received prenatal breastfeeding training $(67.8 \%)$ than among mothers who did not receive that training (28\%) [32]. In the present study, $94.7 \%$ of the experimental-group mothers practiced exclusive breastfeeding. This rate was $26.3 \%$ in the control group $(p<0.05)$. Similar to those in the literature, the first 6-month exclusive breastfeeding rates in the experimental group were higher.

In the initiation and continuation of breastfeeding, support particularly from the spouse has a positive effect on a woman's breastfeeding experience [13]. In several studies it has been determined that mothers are more determined to initiate, continue breastfeeding and tackle problems better if they receive support from their spouses for breastfeeding [33-35]. In Şencan's study, it was determined that mothers who received their husbands support for breastfeeding breastfed their babies longer than did mothers who did not receive support (14.8 and 9.6 months respectively) [36]. In the present study, during the prenatal period when the fathers in the experimental and control groups were asked whether they wanted their wives to breastfeed, they all stated that they wanted. While all the experimentalgroup mothers spoke with their spouses about breastfeeding, this rate was only $5.3 \%$ in the control group. Levels of support regarding exclusive breastfeeding and continuation of breastfeeding up to two years of age were high too. These results are important because they indicate that husbands' decision about and support for breastfeeding encouraged their wives to practice exclusive breastfeeding in the first six months of life.

On the other hand in several studies, it has been determined that training and counseling given to husbands on breastfeeding increase rates of initiation and continuation of breastfeeding during the first six months [3,37-40]. Similar to those studies, higher rates of exclusive breastfeeding behaviors observed in the present study in the experimental group suggest that training and counselling given to husbands on breastfeeding led to increases in the mothers' exclusive breastfeeding practices.

In other studies in the literature, it has also been determined that mothers who breastfeed their babies in the first hour postpartum have more successful lactation process [13,21]. According to TDHS 2013, only $50 \%$ of the babies are breastfed within the first hour after birth [5]. In a study conducted in Turkey, it was determined that $54.9 \%$ of the mothers who were given prenatal care breastfed their babies within the first hour after birth [41]. In the present study, while all the experimental-group mothers breastfed their babies within the first thirty minutes postpartum, only half of the control-group mothers started breastfeeding within one hour after birth $(\mathrm{P}<0.05)$. Hospital policies, support by health professionals who give postpartum healthcare to mothers and mothers' being informed about these issues play an important part in the initiation of breastfeeding process within the first hour after birth.

Frequency and duration of breastfeeding are important factors in the successful continuation of the breastfeeding process [42]. Correct frequency and duration of breastfeeding not only lead to the milk letdown reflex and emptying of the milk ducts, but also play an important role in providing a healthy diet for the baby. According to TDHS 2008, $95 \%$ of the infants younger than six months old were breastfed six or more times within the 24 hours [4]. In a study conducted by Özer et al., $60.2 \%$ of the mothers breastfed their babies at an appropriate frequency [43]. In the present study, the rate of the experimental-group mothers who breastfed their babies within the first 48 hours and sixth months postpartum at two-hour intervals was very high. The rate of those who continued to breastfeed for at least 15-20 minutes was also high in the experimental group. Thus, the experimental-group mothers who managed effective breastfeeding may not have needed to give complementary food.

In her study, Karaçam reported that frequent crying of babies worried mothers that the baby did not get enough milk, which probably was the most important factor urging mothers to give complementary food [44]. In their study, O'campo et al. evaluated the variables affecting breastfeeding and found that the most important factor affecting breastfeeding was the mothers' perception of Breastfeeding SelfEfficacy [45]. In the present study, the experimental-group mothers' Breastfeeding Self-Efficacy scores were higher. The difference between the two groups was significant.

In a study, the cause for the early weaning of breastfeeding among the mothers was often lack of breast milk [13]. In the present study, the IMDAT form was used by the mothers to assess the amount of milk babies received. Comparison of the IMDAT form revealed a significant difference between mean scores of the two groups.

Development and maintenance of infant-mother attachment, the first contact between the mother and baby and breastfeeding are all important especially if they occur in a short time after birth. A mother's breastfeeding the infant immediately after birth brings about the first contact, and correct breastfeeding leads to infants to form secure attachment [46]. In Yalçın's study, no statistically significant difference was determined between attachment scores in terms of the mothers' distribution regarding their breastfeeding behaviors [47]. In several studies, attachment scores of the mothers who exclusively breastfeed have been found higher [48-50]. In the present study, the experimentalgroup mothers exclusively breastfed their babies longer during the first 6 months. The difference between the two groups in terms of the mean maternal attachment scores was significant.

Fathers begin to accept the role of a father after birth. Fathers can develop a sense of caring and love for the baby only after birth. A father can develop an emotional relationship with the baby only after establishing a physical contact [51]. In Zimerman's study, attachment scores of fathers whose wives practiced exclusive breastfeeding longer were found to be higher [50]. In the present study, the experimentalgroup fathers' babies were exclusively breastfed longer within the first 6 
months. The difference between the two groups in terms of the fathers' mean attachment scores was significant.

\section{Conclusions}

It was determined that rates for the early initiation of breastfeeding, rates for exclusive breastfeeding within the first six months and mean scale scores increased when parents were given training and counseling on breastfeeding during the prenatal period and the first six months postpartum. It is recommended that both mothers and fathers be given training and counseling on breastfeeding during the prenatal period by healthcare professionals and be supported after birth.

\section{Declaration of conflicting interests}

The approval to conduct the study was obtained from the Clinical Research Ethics Committee of Cumhuriyet University. The authors declared that they have no conflict of interest.

\section{Funding}

This research was financially supported by the Scientific Research Committee of Cumhuriyet University, Turkey (CUBAP).

\section{References}

1. WHO (2014) Exclusive breastfeeding, Nutrition.

2. Policy Statement (2012) Breastfeeding and the Use of Human Milk, PEDIATRICS.

3. Türkiye Nüfusve Saglik Arastirmasi (TNSA) (2003)

4. Türkiye Nüfusve Saglik Arastirmasi (TNSA) (2008)

5. Türkiye Nüfusve Saglik Arastirmasi (TNSA) (2013)

6. Öztek Z, Kubilay G (1997) Toplum Sagligi Hemsireligi, Ankara, Somgür Yayincilik.

7. Quinn A, Haller S (1997) Breastfeeding Incidence After Early Discharge Factors Influencing Breastfeeding Cessation Journal Obstetric and Gynecologic Neonatal Nursing 26: 289-294.

8. Arora S, McJunkin C, Wehrer J, Kuhn P (2000) Major factors influencing breastfeeding rates: Mother's perception of father's attitude and milk supply. Pediatrics 106: E67. [Crossref]

9. Erdogan A (2004) Çocugun Psikososyal Gelisiminde Babanin Rolü, Yeni Symposium 42: $147-153$.

10. Akan N, Ilk (1995) AydaSadece Anne Sütü Ile Beslenmede Hemsire Etkinliginin Incelenmesi [dissertation], Hacettepe Üniversitesi Saglik Bilimleri Enstitüsü Hemsirelik Programi, Ankara.

11. Eker A, Yurdagül M (2006) Annelerin Bebek Beslenmesive Emzirmeye Iliskin Bilgive Uygulamalari, STED (Sürekli Tip Egitimi Dergisi), 15: 158-163.

12. Scott JA, Binns CW, Oddy WH, Graham KI (2006) Predictors of breastfeeding duration: evidence from a cohort study. Pediatrics 117: e646-655. [Crossref]

13. Gözükara F (2012) Ebeveynlere Emzirmeye Yönelik Verilen Egitim Ve Danismanlik Hizmetlerinin Emzirme Davranisina Etkisi [dissertation], Hacettepe Üniversitesi Saglik Bilimleri Enstitüsü Dogum-Kadin Hastaliklari Hemsireligi Programi, Ankara.

14. Yigit F, Arslan H (2008) Bebegin Aldigi Anne Sütü Miktarinin DegerlendirilmesiIçin Bir Araç: IMDAT, Zeynep Kamil Tip Bülteni 39:77-84.

15. Tokat MA (2009) Antenatal Dönemde Verilen Egitimin Annelerin Emzirme ÖzYeterlilik Algisinave Emzirme Basarisina Etkisi [dissertation], Dokuz Eylül Üniversitesi Saglik Bilimleri Enstitüsü, Dogumve Kadin Hastaliklari Hemsireligi, Izmir.

16. Müller ME (1994) A questionnaire to measure mother-to-infant attachment. J Nurs Meas 2: 129-141. [Crossref]

17. Beck CT (1998) A review of research instruments for use during the postpartum period, Maternal Child Nursing (MCN) 123: 255-6.

18. Condon JT, Corkindalea CJ, Boyce P (2008) Assessment of postnatal paternal-infant attachment: development of a questionnaire instrument. Journal of Reproductive and Infant Psychology 26: 195-210.
19. Güleç D (2010) Baba Bebek Baglanma Ölçeginin Türk Toplumunda Geçerlikve Güvenirliginin Incelenmesi [dissertation], Ege Üniversitesi Saglik Bilimleri Enstitüsü, Kadin Sagligive Hastaliklari Hemsireligi ABD, Izmir.

20. WHO (2014) World Health Statistics.

21. Ünsal H, Altihan F, Özkan H, Targan S, Hassoy H (2005) Toplumda annesütüvermeegilimivebunaetkiedenfaktörler Çocuk Sagligive Hastaliklari Dergisi 48: 226-233.

22. Artieta-Pinedo I, Paz-Pascual C, Grandes G, Bacigalupe A, Payo J, et al. (2013) Antenatal education and breastfeeding in a cohort of primiparas. J Adv Nurs 69: 16071617.[Crossref]

23. Howel D, Ball H (2013) Association between length of exclusive breastfeeding and subsequent breastfeeding continuation. J Hum Lact 29: 579-585.[Crossref]

24. Tanner-Smith EE, Steinka-Fry KT, Lipsey MW (2013) Effects of CenteringPregnancy group prenatal care on breastfeeding outcomes. J Midwifery Womens Health 58: 389 395. [Crossref]

25. Üstüner F, Bodur S (2009) Bebeklerde ayliki zlem ve hemsire tarafindan anneninpe ki stirici emzirme egitim ile ilk alti ay yalnizca anne sütü verme arasindaki iliski, Genel Tip Derg, 19: 25-32, 2009.

26. Lawrence RA (2011) Lawrence, R.M, Breastfeeding. A Guide For The Medical Profession, Mosby, Elsevier.

27. Aykut M (2010) Toplum Beslenmesi, Halk Sagligi Genel Bilgileri'nde. Eds. Özturk Y, Günay O. Kayseri, Erciyes Üniversitesi Yayinlari 1247- 417.

28. WHO (2014) Evidence for the ten steps to successful breastfeeding.

29. Artieta-Pinedo I, Paz-Pascual C, Grandes G, Bacigalupe A, Payo J, et al. (2013) Antenatal education and breastfeeding in a cohort of primiparas. J Adv Nurs 69: 16071617.[Crossref]

30. Lumbiganon P, Martis R, Laopaiboon M, Festin MR (2012) Antenatal Breastfeeding Education ForIncreasing Breastfeeding Duration (Review), The Cochrane Collaboration.

31. Merewood A (2014) Prenatal education: timing it right. J Hum Lact30: 133.[Crossref]

32. Onbasi S, Duran R, Çiftdemir NA, Vatansever Ü, Acunas B (2011) Dogum öncesi anne adaylari naverilen emzirme ve anne sütü egitiminin emzirme davranislari üzerine etkisi, Türk Pediatri Arsivi 46:75-80.

33. Februhartanty J, Bardosono S, Septiaril AM (2006) Problems During Lactation are Associated with Exclusive Breastfeeding in DKI Jakarta Province: Father's Potential Roles in Helping to Manage These Problems Mal. J. Nutr 12: 167-180.

34. Küçükosmanoglu E, Acar Y, Altinel N, Kaçar A (2001) Dogacak Bebegin Emzirilmesi Konusunda Baba AdaylarininYaklasimi, Çocuk Sagligive Hastaliklari Dergisi 44: 349354

35. Sharma M, Petosa R (1997) Impact of expectant fathers in breast-feeding decisions. $J$ Am Diet Assoc 97: 1311-1313.[Crossref]

36. Sencan I (2008) Anne Sütü Ile Beslenme Süresine Etki Eden Faktörlerin Arastirilmasi [dissertation], Fatih Üniversitesi, Ankara.

37. Ito J, Fujiwara T, Barr RG (2013) Is paternal infant care associated with breastfeeding? A population-based study in Japan. J Hum Lact 29: 491-499 .[Crossref]

38. Mitchell-Box KM, Braun KL (2013) Impact of male-partner-focused interventions on breastfeeding initiation, exclusivity, and continuation. J Hum Lact 29: 473-479. [Crossref]

39. Mitchell-Box K, Braun KL, Hurwitz EL, Hayes DK (2013) Breastfeeding attitudes association between maternal and male partner attitudes and breastfeeding intent. Breastfeed Med 8: 368-373. [Crossref]

40. Maycock B, Binns CW, Dhaliwal S, Tohotoa J, Hauck Y, et al. (2013) Education and support for fathers improves breastfeeding rates: a randomized controlled trial J Hum Lact 29: 484-490. [Crossref]

41. Çetin F, Günes G, Karaoglu L, Üstün Y, Turgut Özal (2005) Tip Merkezinde Dogum Yapan Annelerin Dogum Öncesi Bakim Alma Ve Emzirmeye Baslama Durumlari Ve Etkileyen Faktörler, Inönü Üniversitesi Tip Fakültesi Dergisi.

42. Taskin L (2007) Normal Yenidoganin Fizyolojisive Bakimi. L. Taskin Eds. Dogumve Kadin Sagligi Hemsireligi 241 Sistem Ofset Matbaacilik, Ankara.

43. Özer A, Tas F, Ekerbiçer HÇ (2010) Aylik Bebegi Olan Annelerin Anne Sütüve Emzirme Konusundaki Bilgive Davranislari TAF Preventive Medicine Bulletin 9: 315 320. 
Yurtsal ZB (2015) The effects of antenatal parental breastfeeding education and counseling on the duration of breastfeeding, and maternal and paternal attachment

44. Karaçam Z (2008) Factors affecting exclusive breastfeeding of healthy babies aged zero to four months: a community-based study of Turkish women J Clin Nurs 17: 341-349. [Crossref]

45. O'Campo P, Faden RR, Gielen AC, Wang MC (1992) Prenatal factors associated with breastfeeding duration: recommendations for prenatal interventions. Birth 19: 195201. [Crossref]

46. Sen S (2007) Anneanne-Anne -Bebek Baglanmasinin Incelenmesi [dissertation], Ege Üniversitesi Saglik Bilimleri Enstitüsü, Izmir.

47. Yalçin JY (2010) Anne Bebek Arasindaki Baglanma Olgusunun Infantil Kolik Ile Iliskisi [dissertation], Mersin Üniversitesi Saglik Bilimleri Enstitüsü Hemsirelik Anabilimdali, Mersin.
48. Scharfe E (2012) Maternal attachment representations and initiation and duration of breastfeeding. J Hum Lact 28: 218-225. [Crossref]

49. Labarère J, Gelbert-Baudino N, Laborde L, Baudino F, Durand M, et al. (2012) Determinants of 6-month maternal satisfaction with breastfeeding experience in a multicenter prospective cohort study J Hum Lact 28: 203-210. [Crossref]

50. Zimerman A (1992) Maternal- and paternal-fetal attachment in relation to breastfeeding Dissertations \& Theses (PQDT)pg. n/a York University, Ontario.

51. Kavlak O, Sirin A (2007) Maternal And Paternal Attachment And The Role Of Nurse Journal of Ege University School of Nursing 23: 183-194.

Copyright: $\odot 2015$ Yurtsal ZB. This is an open-access article distributed under the terms of the Creative Commons Attribution License, which permits unrestricted use, distribution, and reproduction in any medium, provided the original author and source are credited. 